\title{
Decortication as an Option for Empyema Thoracis
}

\author{
Farhan Ahmed Majeed11, Usama Zafar1, Sohail Saqib Chatha², Ahmad Ali¹ and Ahmed Raza1 \\ IDepartment of Thoracic Surgery, Combined Military Hospital, Lahore, Pakistan \\ 2Department of Surgery, PAF Hospital, Lahore, Pakistan
}

\begin{abstract}
Objective: To analyse the outcome and morbidity associated with decortication in empyema thoracis. Study Design: A case series.

Place and Duration of Study: Departments of Surgery, Combined Military Hospitals (CMH) of Rawalpindi, Quetta and Lahore, from January 2006 to March 2018.

Methodology: This is a retrospective study of 812 cases of open and VATS (video-assisted thoracic surgery) decortication for empyema thoracis, operated by the same consultants. Only patients with established empyema were included. Those who were unfit for one-lung ventilation, undergoing local anesthesia procedures like rib resection, clagget window or tube windows, with clotted hemothorax and malignant pathology were excluded. Posterolateral serratus sparing thoracotomy was used in open decortications. Multiportal or uniport VATS was employed for video-assisted thoracoscopic decortications (VATD). Histopathology and microbiological sampling was also done in all cases.

Results: There were $537(66.1 \%)$ males and 275 (33.9\%) females. Age ranged from 1 to 80 years with a mean of 37 years. Open decortication was done in $650(80 \%)$, standard decortication with posterolateral thoracotomy in $458(56.4 \%)$, minithoracotomy was done in $69(8.4 \%)$ patients with loculated empyema, thoracotomy and open decortication with conventional thoracoplasty was done in 21 patients. Twenty-two patients required open decortications with tailored thoracoplasty and muscle flap. Open decortication with intercostal muscle (ICM) flap or primary closure of bronchopleural fistula was performed in 55 patients. VATD was done in 162 cases, out of which 120 were early empyema, and 42 were of chronic empyema; of which 22 required a further utility thoracotomy. Decortication with lung resection and muscle flap reinforcement to bronchial stump was done in 25 patients. Blood transfusion was required in 331 (40.7\%). Twenty-six $(3.4 \%)$ patients developed residual space and collection requiring intervention; and $384(47.3 \%)$ patients had a histopathology diagnostic for tuberculosis. There were $11(1.3 \%)$ deaths.

Conclusion: Open decortication is still one of the preferred procedures in developing countries. VATD is also increasingly utilised for empyema.
\end{abstract}

Key Words: Empyema, Decortications, VATS, Thoracotomy, Tuberculosis.

How to cite this article: Majeed FA, Zafar U, Chatha SS, Ali A, Raza A. Decortication as an option for empyema thoracis. J Coll Physicians Surg Pak 2020; 30(3):313-317.

\section{INTRODUCTION}

Empyema, pus in the pleural cavity, often occurs as a result of pneumonia. However, in developing countries tuberculous etiology of empyema is more common.1,2 Empyema develops through three phases which include exudative, fibrinopurulent and organising phase. ${ }^{3}$ During early stage of empyema antimicrobial treatment and chest tube drainage constitute effective treatment option for space sterilisation. However, in advanced stages like fibrinopurulent and organising phases, this combination of antibiotic treatment and chest tube drainage fails in most of the cases, due to loculation of fluid and thick pleural peel with failure of lung to expand. Decortication is needed which involves either VATS or open thoracotomy. Decortication is considered as a method of

Correspondence to: Dr. Usama Zafar, Department of Thoracic Surgery, Combined Military Hospital, Lahore, Pakistan

E-mail:usamazafar@gmail.com

Received: November 12, 2018; Revised: August 06, 2019;

Accepted: September 05, 2019 choice when there is thick inflammatory coat over the lung which prevents the lung to expand. ${ }^{4}$ In the last few decades, open decortication is considered as the most favoured approach due to shorter operative duration, better vision, and better lung re-expansion. ${ }^{5}$ VATD has postoperative advantages, including less pain, morbidities, complications, and mortality. 6 Despite higher rates of conversion to open decortication and technical difficulties during the surgical procedure, VATS drainage has clearly showed better results when applied at the earlier stages of empyema, ${ }^{7}$ especially during the fibrinopurulent stage. A systematic review concluded that VATD has superior outcomes in the treatment of empyema thoracis. ${ }^{8}$ However, in developing countries where TB is more prevalent and associated with development of empyema and bronchopleural fistula, open decortication is still more practised than VATD. Moreover late presentation of the disease in developing countries due to late referrals as a result of financial and logistic constraints also makes open decortications more favourable. 
The rationale of this study is to emphasise the importance of open and VATS decortication as valid and preferred treatment option in thoracic surgery centres of developing countries, highlighting the importance of tailoring treatment strategy according to stage and pathology of the underlying lung parenchyma and encouraging early surgical referrals. The objective of this study was to analyse the outcome and morbidity associated with decortication in empyema thoracis.

\section{METHODOLOGY}

Record of 812 thoracic empyema patients treated in $\mathrm{CMH}$ Rawalpindi, Quetta and Lahore over a period of 12 years between Jan 2006 to Mar 2018, was analysed. Anonymity of the patients was ensured by removing their identification numbers, names and pictures. Data regarding clinical profile, stage of the disease, cause of empyema, type of procedure performed and complications was recorded.

Patients with established empyema were included in the study. Patients unfit for one lung ventilation, undergoing rib resection, clagget or tube windows were excluded. Patient with clotted hemothorax and malignancy were also excluded. VATD or open thoracotomy was done depending upon the stage of the empyema and condition of underlying lung parenchyma for space obliteration. Various parameters such as hospital stay, postop X-ray findings, and blood transfusions were noted. Bacterial culture of pus and histopathology of the tissues followed.

\section{RESULTS}

A total of 812 patients were diagnosed as cases of thoracic empyema. Patients were aged between 1 to 80 years. Three hundred and sixty-nine (45\%) belong to young age group i.e. $21-40$ years followed by $278(34.2 \%)$ in 41-60 years, $118(14.5 \%)$ in 1-20 years and $47(5.8 \%)$ in 61-80 years age groups. Further demographics are given in Table I. Five hundred and thirty-seven (66.1\%) were males while $275(33.9 \%)$ were females. Five hundred and ninety-eight $(73.6 \%)$ stayed for $<5$ days, while $214(26.4 \%)$ had hospital stay of $\geq 5$ days. Amongst patients undergoing VATS (n162), only 24 (14.9\%) stayed in the hospital for $\geq 5$ days while stay of the remaining $138(85.1 \%)$ patients was $<5$ days.

Most common cause of empyema thoraces was tuberculosis $(47.3 \%)$, followed by pneumonia $(30.3 \%)$, posttrauma $(11.8 \%)$, post-surgery $(3.9 \%)$, and miscellaneous $(6.7 \%)$.

Six hundred and fifty (80\%) patients underwent open thoracotomy, while $162(20 \%)$ underwent VATD. In order to systematise the management strategy, an algorithm was designed for chronic empyema (Figure 1). Patients were broadly categorised into two main categories: category A consisted of patients of empyema without
Table I: Baseline characteristics of study population.

\begin{tabular}{lcc}
\hline Variable & Frequency & Percent \\
\hline Gender & 537 & 66.1 \\
Male & 275 & 33.9 \\
Female & & \\
\hline Year & 75 & 9.2 \\
$2006-2008$ & 65 & 8.0 \\
2010 & 34 & 4.2 \\
2011 & 46 & 5.7 \\
2012 & 220 & 27.1 \\
2013 & 93 & 11.5 \\
2014 & 85 & 10.5 \\
2015 & 83 & 10.2 \\
2016 & 57 & 7.0 \\
2017 & 54 & 6.7 \\
2018 & & \\
\hline Duration of empyema & 119 & 14.7 \\
Early & 693 & 85.3 \\
Late & $<5$ days & 59873.6 \\
\hline Hospital Stay & $>5$ days & 21426.4 \\
\hline & & \\
\hline
\end{tabular}

bronchopleural fistula (BPF), and category B consisted of those with BPF. Category A had generalised disease requiring standard circumhilar decortication and loculated empyema, which were managed with minithorcotomy and decortication. Amongst patients without fistula $\mathrm{BPF}$, open thoracotomy decortication was done in 458 $(56.4 \%)$, minithoracotomy decortication was done in 69 patients $(8.5 \%)$.

Category B patients were further subdivided into those with expandable lungs and those with unexpandable lungs. In empyema with BPF (expandable lung), 29 patients $(3.6 \%)$ underwent decortications with primary repair of BPF followed by repair of BPF with muscle flap in $20(2.5 \%)$, and repair of BPF with lung flap in 6 $(0.7 \%)$. In non-expandable lung group with organised collapse consolidation, decortications with conventional thoracoplasty was done in $21(2.6 \%)$, while $22(2.7 \%)$ underwent decortication and tailored thoracoplasty with muscle flap. In the non-expandable lung group with either cavitatory lung disease, endobronchial symptoms or lung necrosis underwent decortications and lung resection either anatomical or non-anatomical with buttressing muscle flap $(n=25,3.1 \%)$. VATD was done in 162 cases, out of which 120 were of early empyema, 42 were of chronic empyema; and out of these chronic empyema, 22 required a utility thoracotomy with VATS.

Among open surgical procedures $(n=650)$ serratus sparing was done in $562(86.4 \%)$, while total muscle sparing was done in $80(12.3 \%)$ no muscle sparing in 8 . Empyema fluid, culture yielded no growth in 355 $(43.7 \%)$, followed by gram positive in $201(24.8 \%)$, gram negative in $185(22.8 \%)$, anaerobes in $66(8.1 \%)$ cases, while growth of fungus was seen in $5(0.7 \%)$.

Histopathology showed chronic caseating necrosis in $223(27.5 \%)$ while granulomas were seen in 189 


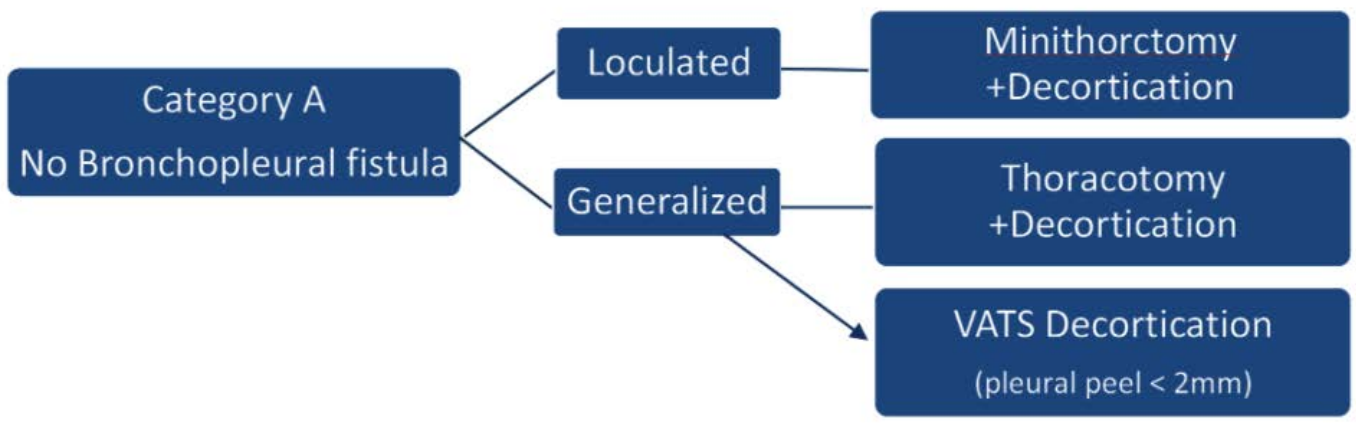

- Standard circumhilar decortication

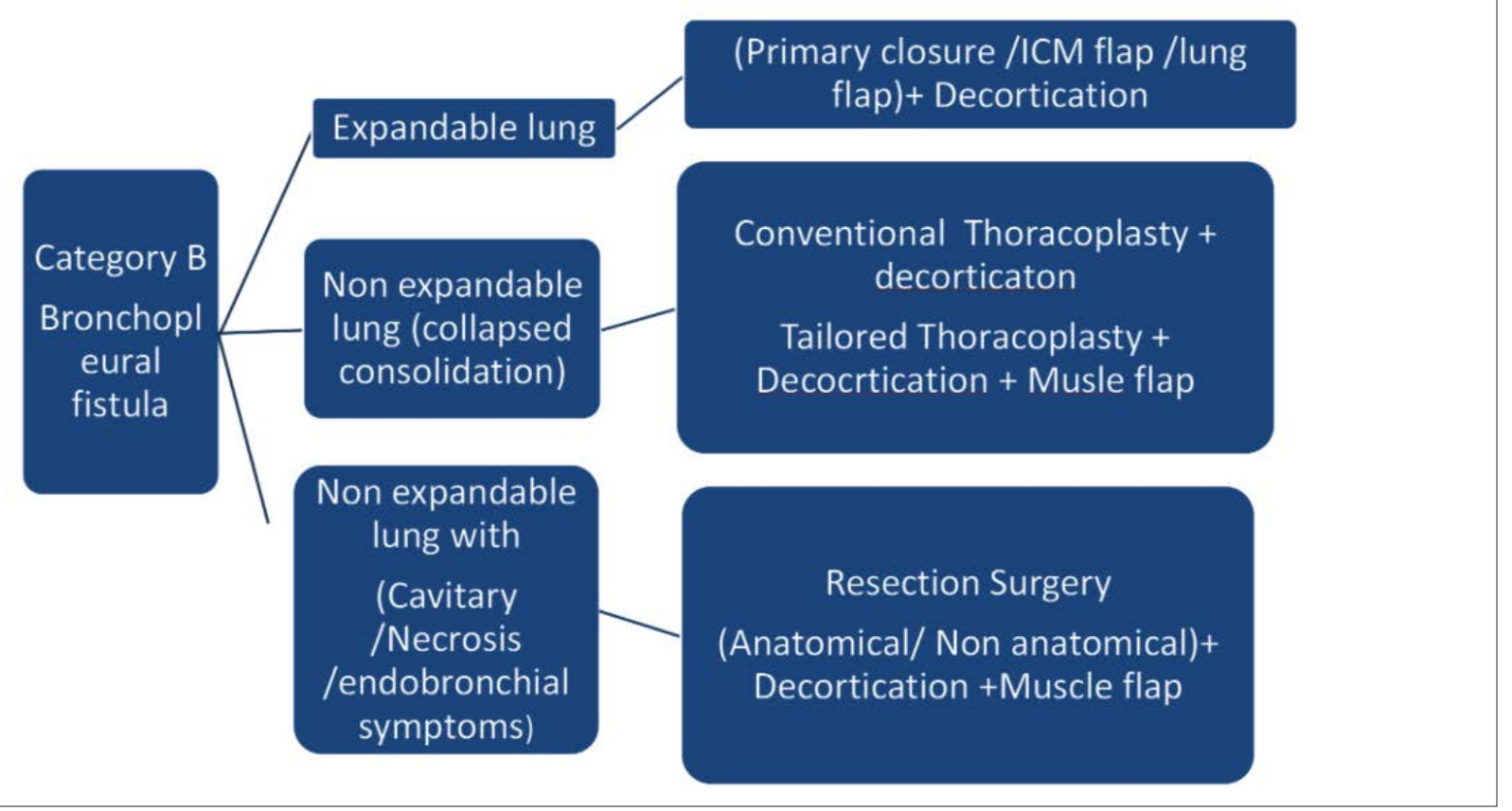

Figure 1: Algorithm for management of empyema thoraces.

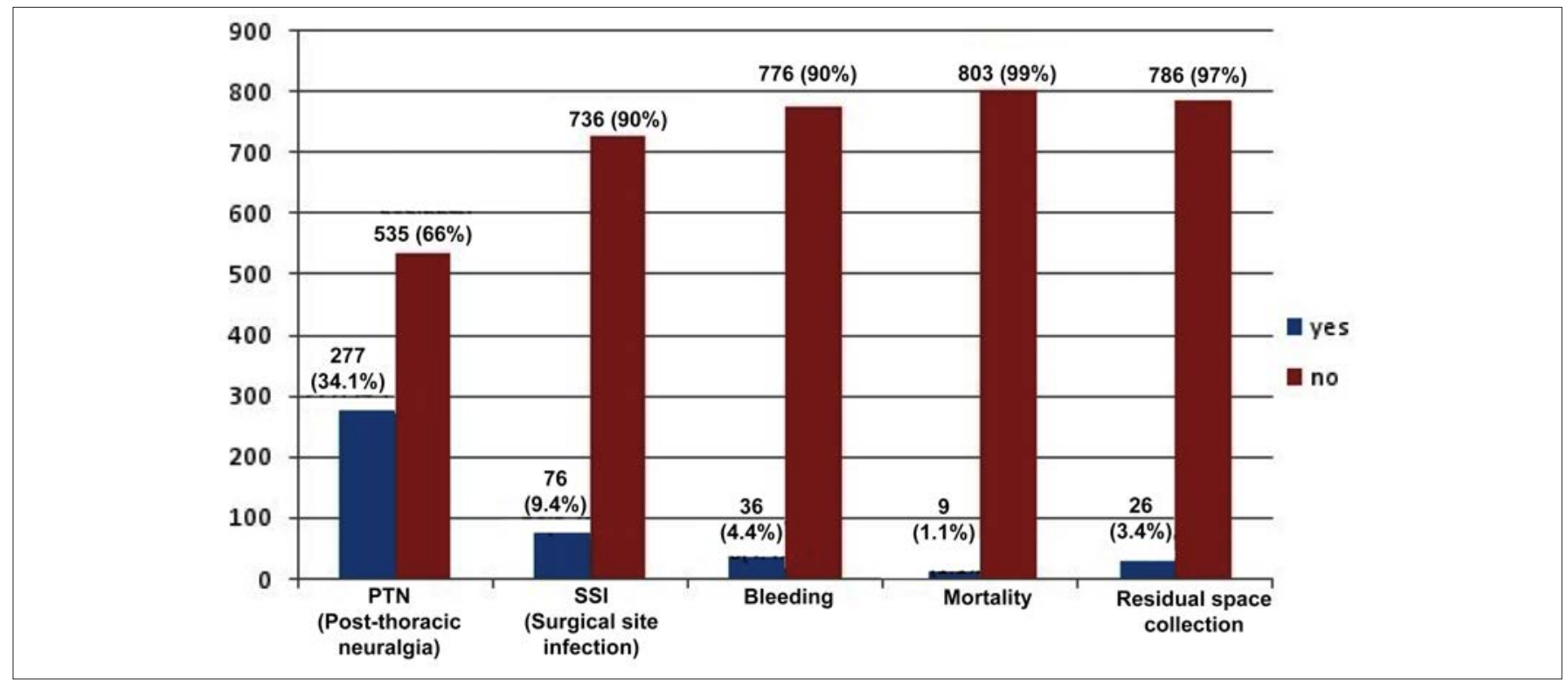

Figure 2: Complications break-up. 
(23.3\%). Blood transfusion was required in 331 (40.7\%). One unit of blood was transfused in 197, 2 units transfused in 108 patients, 3 units in 19 patients, 4 units in 6 patients while one patient was transfered 6 units of whole blood. Complications are given in Figure 2. There are 9 deaths $(1.1 \%)$ with none among the VATS cohort.

\section{DISCUSSION}

Empyema thoracis is a worldwide problem affecting all age groups. A major problem in the management of thoracic empyema is formation of fibrinous adhesions in the visceral and parietal pleura, which can lead to entrapment of lung, renders it difficult to drain the pleural space and failing in reexpansion of lung by antibiotics and thoracocentesis alone. Various studies have shown that early decortication is safe and leads to rapid resolution of symptoms along with radiological resolution and reducing hospital stay as also shown in our study where average stay of patients $75 \%$ was less than 5 days. These findings have also been confirmed by other studies such as done by Hilliard and colleagues, where mean hospital stay was of four days only. 9,10

Majority of the empyemas develop due to pneumonia with various studies showing an incidence of $40-57 \%$ in patients hospitalised with bacterial pneumonia.11,12 However, empyema caused by TB is more prevalent among TB-endemic nations, 12,13 as was the case in this study, where TB was responsible for $47.3 \%$ of the cases followed by pneumonia in $30.3 \%$ of cases. Hence, in TBendemic areas, empyema cases must be investigated for tuberculous etiology. Microbial culture isolates obtained in our study are similar to other series of adult empyema thoracis with gram positive organisms $(24.8 \%)$ being the most common isolate. ${ }^{13}$

An aggressive surgical approach was adopted in majority of the cases with empyema thoracis. Our strategy of early surgical management in patients of loculated pleural effusions/empyema has been verified in various other studies. ${ }^{14}$ Various surgical techniques have been used in the treatment of empyema including open decortication, VATD, thoracoplasty with muscle or lung flap, and resections. In this study, standard decortication was the major procedure performed in $56.4 \%$ patients, followed by VATD in $20.0 \%$. Decortication is considered as the method of choice when the underlying lung is unable to expand due to the thick inflammatory coat and the patient is fit for major surgery, ${ }^{15}$ as was the case in this study. Majority of the presently reported patients were referred at a late stage after initial treatment with repeat thoracentesis or closed thoracostomy and were, therefore, relatively chronic with multiple pleural adhesions as has also been mentioned in another series by Ridley. ${ }^{16}$ Success rate of VATD mainly depends on presentation at earlier stage with majority of patients in the organising phase have higher failure rate. ${ }^{17}$ Due to chronicity of empyema, only $20.0 \%$ patients in our study underwent VATD. Thoracoplasty involves remodelling of the osteomuscular wall of the thoracic cage in order to control the underlying inflammatory process; but is rarely performed these days. ${ }^{18}$ In this study, 43 patients (5.3\%) underwent thoracoplasty alone and with muscle flap.

Treatment of empyema with BPF, remains a challenge. In our study different methods such as primary repair of BPF and repair with muscle or lung flap were utilised for the treatment. Intrathoracic transposition of extrathoracic skeletal muscles is not a new concept. In 1949, Maier described the use of pectoral myoplasty for obliteration of empyema cavities after thoracoplasty. ${ }^{19}$

Empyema is a disease with considerable mortality, reaching upto $20 \%$ in adults when treatment is delayed. However early surgical treatment whether through VATS or open decortication, is associated with favourable outcome. Overall mortality is considered low for VATD as compared to open decortication, being $5.4 \%$ and $6.3 \%$, respectively, in few studies. 20 However, in this study there were 11 deaths $(1.1 \%)$, none amongst the VATD. Among the complications encountered, PTN being the most common followed by surgical site infection and bleed. Based on this study, it is imperative that early aggressive surgical treatment of pleural empyema whether by open technique or VATD, holds the key for reducing morbidity and mortality. Different surgical options can be utilised and tailored depending upon the stage of empyema and condition of the patient.

This study, being a retrospective one and having selective and limited study population and clinical settings, may have led to selection bias. Pakistan being a developing country, tuberculous empyema was more common as compared to para pneumonic collections. Here patients usually present at later stage; hence, surgical options utilised in this study are more representative of advanced and complex forms of empyema.

\section{CONCLUSION}

Decortication for chronic empyema remains the commonest surgical procedure in thoracic surgery unit in developing countries because of late referrals, high prevalence of tuberculosis, financial and logistic issues in health care management. Thoracic surgery residents of developing countries should be proficient in management of empyema thoracis due to high volume of inflammatory and tuberculous lung pathologies there.

\section{ETHICAL APPROVAL:}

Ethical approvals were obtained prior to initiation of the research work from the Ethical Committee of $\mathrm{CMH}$, Lahore.

\section{PATIENTS' CONSENT:}

Informed consents were taken from all patients. 


\section{CONFLICT OF INTEREST:}

Authors declared no conflict of interest.

\section{AUTHORS' CONTRIBUTION:}

FAM: Data collection, data analysis, abstract writing, proofreading.

UZ: Data analysis, data collection.

SC: Discussion and introduction writing, statistical analysis.

AA: Picture contribution, data analysis.

AR: Reference writing, result compilation.

\section{REFERENCES}

1. Sharma SK, Mohan A. Extrapulmonary tuberculosis. Indian J Med Res 2004; 120:316-53.

2. Al-Kattan KM. Management of tuberculous empyema. Eur $J$ Cardiothorac Surg 2000; 17:251-4.

3. American Thoracic Society. Management of nontuberculous empyema. Am Rev Respir Dis 1962; 85:935-6.

4. Anstadt MP, Guill CK, Ferguson ER. Surgical versus nonsurgical treatment of empyema thoracis: An outcomes analysis. Am J Med Sci 2003; 326:9-14.

5. Luh SP, Hsu GJ, Cheng-Ren C. Complicated parapneumonic effusion and empyema: Pleural decortication and videoassisted thoracic surgery. Curr Infect Dis Rep 2008; 10:236-40.

6. Tong BC, Hanna J, Toloza EM. Outcomes of videoassisted thoracoscopic decortication. Ann Thorac Surg 2010; 89:220-5.

7. Chung J, Sung L. Optimal timing of thoracoscopic drainage and decortication for empyema. Ann Thorac Surg 2014; 97:224-9.

8. Chambers A, Routledge T, Dunning J. Is video-assisted thoracoscopic surgical decortication superior to open surgery in the management of adults with primary empyema? Interact Cardiovasc Thorac Surg 2010; 11:171-7.
9. Carey JA, Hamilton JRL, Spencer DA. Empyema thoracis: A role for open thoracotomy and decortication. Arch Dis Child 1988; 79:510-13.

10. Hilliard TN, Henderson AJ, Langton Hewer SC. Management of parapneumonic effusion and empyema. Arch Dis Child 2003; 88:915-7.

11. Koegelenberg CFN, Diacon AH, Bolliger CT. Parapneumonic pleural effusion and empyema. Respiration 2008; 75:241-50.

12. Taryle DA, Potts DE, Sahn SA. The incidence and clinical correlates of parapneumonic effusions in pneumococcal pneumonia. Chest 1978; 74:170-3.

13. Lemmer JH, Botham MJ, Orringer MB. Modern management of adult thoracic empyema. J Thorac Cardiovasc Surg 1985; 90 349-55.

14. Bilgin M, Akcali Y, Oguzkaya F. Benefits of early aggressive management of empyema thoracis. ANZ J Surg 2006; 76:120-2.

15. Anstadt MP, Guill CK, Ferguson ER. Surgical versus nonsurgical treatment of empyema thoracis: An outcomes analysis. Am J Med Sci 2003; 326:9-14.

16. Ridley PD, Braimbridge MV. Ann Thorac Surg 1991; 51:461-4

17. Luh SP, Chou MC, Wang LS, Chen JY, Tsai TP. Video-assisted thoracoscopic surgery in the treatment of complicated parapneumonic effusions orempyemas. Chest 2005; 127: 1427-32.

18. Molnar TF. Current surgical treatment of thoracic empyema in adults. Eur J Cardiothorac Surg 2007; 32:422-30.

19. Maier HC, Luomanen RKJ. Pectoral myoplasty for closure of residual empyema cavity and bronchial fistula. Surgery 1949; 256214.

20. Semenkovich TR, Olsen MA. Ann Thorac Surg 2018; 105: 1589-96. 